\title{
Primary ciliary dyskinesia: keep it on your radar
}

\author{
Margaret Rosenfeld, ${ }^{1}$ Lawrence E Ostrowski, ${ }^{2,3}$ Maimoona A Zariwala ${ }^{4}$
}

Primary ciliary dyskinesia (PCD) is a rare disorder of mucociliary clearance resulting in chronic oto-sinopulmonary disease. While the prevalence worldwide is estimated to be 1:10000-1:15 000, it may be much higher in certain communities, especially where consanguinity is more prevalent. In the British South Asian community, for example, the prevalence has been estimated at 1:2450, similar to that of cystic fibrosis among caucasians. ${ }^{1}$

In Thorax, Shoemark and colleagues ${ }^{2}$ describe PCD in a highly consanguineous UK South Asian community, due to homozygous missense variants in CCDC103, usually resulting in the loss of both outer and inner dynein arms. The investigators identified 86 patients of South Asian (primarily Pakistani) descent with clinical signs and symptoms of PCD from the UK National PCD Diagnostic and Management Services. These individuals had a compatible clinical phenotype, but diagnosis was complicated by the fact that many of the standard diagnostic tests for PCD had yielded variable and frequently normal results, including nasal nitric oxide, ciliary beat frequency and ciliary ultrastructure by transmission electron microscopy. Ultimately, next-generation sequencing confirmed a homozygous pathogenic variant, p.His154Pro, in CCDC103 in 16 (19\%) of these 86 cases, from 12 independent families.

From a clinical perspective, this report by Shoemark and colleagues ${ }^{2}$ highlights two important issues in the diagnosis of PCD. The first is that the prevalence of PCD may be higher than previously appreciated in certain populations, particularly in highly consanguineous communities. Indeed, in this report, all 16 patients with homozygous CCDC103 p.His154Pro mutations were children of

'Department of Pediatrics, University of Washington School of Medicine, Seattle, Washington, USA

${ }^{2}$ Department of Medicine, University of North Carolina, Chapel Hill, North Carolina, USA

${ }^{3}$ Marsico Lung Institute, Cystic Fibrosis Research Center, University of North Carolina, Chapel Hill, North Carolina ${ }^{4}$ Department of Pathology and Laboratory Medicine, University of North Carolina, Chapel Hill, North Carolina, USA

Correspondence to Dr Margaret Rosenfeld, Department of Pediatrics, University of Washington School of Medicine, Seattle, WA 98195, USA; margaret. rosenfeld@seattlechildrens.org consanguineous parents. While this UK South Asian PCD cohort was enriched for the CCDC103 p.His154Pro mutation, a number of other PCD-causing mutations were also present, including CCDC40, DNAAF1, HEATR2, LRRC6, ZMYND10 and RSPH4A. A high prevalence of PCD with genetic heterogeneity has similarly been reported among the close-knit, socially isolated, highly consanguineous US Amish and Mennonite communities, due to a founder pathogenic variant in DNAH5 and HEATR2. ${ }^{3}$

In the current study, the allele frequency of p.His154Pro from the ethnically matched control cohort was 0.00195 (six of 3084 alleles). In the gnomAD public database (gnomad.broadinstitute. org/), composed of $>277000$ alleles from diverse population cohorts, the allele frequency of this missense variant is reported as 0.00123 and all alleles were present in a heterozygous state. Interestingly, on reviewing individual cohorts in gnomAD, the observed allele frequency among South Asians (0.0032) is approximately double that of European cohort (0.0015). Although, these allele frequencies are seemingly very rare, they are much higher compared with the allele frequencies of loss-of-function pathogenic variants in the same gene. For example, the homozygous pathogenic variant (c.383_384insG (p.Pro129Serfs*25)) in CCDC103 that is associated with $\mathrm{PCD}^{4}$ is not represented in gnomAD. It is also pertinent to mention that p.His145Pro is represented in almost all ethnicities and races (except East Asian) in gnomAD, raising a question as to whether it represents a common 'founder' effect or a mutation 'hotspot?' It may have been useful if the authors had presented the haplotype data extracted from their next-generation sequencing dataset in the cases with the homozygous p.His154Pro variants of South Asian heritage and compared it with the few cases they had of European origin harbouring the same variant in order to better understand the differences in prevalence.

The second clinical issue highlighted by this report is that PCD may be difficult to diagnose, requiring dogged pursuit of multiple diagnostic tests. It is well established that, among persons with a high clinical index of suspicion, no single test can reliably diagnose PCD. Nasal nitric oxide can be normal in certain genetic causes of PCD, including some patients with mutations in RSPH1 in addition to CCDC103. Electron microscopy of ciliary ultrastructure can be normal or inconclusive in up to one-third of patients. ${ }^{5}$ Genetic testing, though improving, cannot currently identify biallelic disease-causing mutations in up to a third of individuals diagnosed with PCD. The 16 patients with PCD harbouring homozygous CCDC103 p.His154Pro variant all possessed classic characteristics of PCD, including situs inversus totalis in 15 (81\%). Yet, seven $(43 \%)$ had a normal nasal nitric oxide on at least one occasion, a test that in general has a high sensitivity and specificity for PCD among persons with a compatible clinical phenotype. ${ }^{6}$ Similarly, nine (56\%) had either normal or inconclusive ciliary ultrastructure by electron microscopy, nine had normal ciliary beat frequency and seven were reported to have a normal ciliary beat pattern. Indeed, five of the 16 did not receive a definitive diagnosis until their genotype was confirmed. This report by Shoemark and colleagues, ${ }^{2}$ thus, highlights the importance of taking a careful history in order to establish a compatible clinical phenotype $\mathrm{p}^{7}$ and then pursuing a multimodal diagnostic approach.

Another interesting aspect of the report is that, unlike many of the PCD-causing mutations to date, the p.His154Pro mutation is not a clear loss-of-function mutation. In fact, the mutation behaves as a hypomorph, and the evidence from this and prior studies ${ }^{4}$ is that the mutant CCDC103 protein is translated and retains at least partial function. This observation is no doubt partially responsible for the variable, sometimes normal, results observed in the diagnostic testing. In the case of CCDC103, patients with pathogenic loss-of function variants have been previously identified, and it is clear that the p.His154Pro variant is pathogenic. However, as more genetic studies are completed and more missense variants are identified in potential PCD-causing genes, it will become increasingly important to determine if the variant is pathogenic. That is why it is important to note that the authors performed an experiment attempting to determine the functional consequence of the p.His154Pro mutation in the human protein. Again, the authors benefited from prior studies, notably those of King and Patel-King, ${ }^{8}$ who characterised the CCDC103 protein from Chlamydomonas and showed the protein forms dimers and higher-order oligomers that bind microtubules with a periodicity 
of $12 \mathrm{~nm}$, potentially helping to define the sites of outer dynein arm binding. In this report, a gel-filtration experiment was performed to compare the wild-type CCDC103 with the pHis154Pro mutant protein. Although the proteins were assayed at very different concentrations (which is critical because oligomerisation is concentration dependent), the authors report a difference between the wild type and the mutant proteins, and the authors should be commended for going beyond characterising the genetics. There is a profound need for additional biochemical studies of this type, not only to determine if a particular missense variant is pathogenic but also to begin to design targeted therapies for treatment. The development of lumacaftor to stabilise the common F508del mutation in cystic fibrosis is a clear example of how understanding the basic biochemical defect can lead to improved treatment. The use of model systems (eg, Chlamydomonas, Xenopus, zebrafish) and/or human cell cultures to define the functional consequences of missense variants, like the p.His154Pro in CCDC103, will be of increasing importance as the development of personalised therapies for patients with PCD accelerates.

Advancesin next-generation sequencing technologies have profoundly increased our understanding of human genetic disorders. However, these advances have also led to the identification of an enormous number of variants of uncertain significance (VUS), interpretation of which remains challenging. The American College of Medical Genetics and Genomics, together with the Association for Molecular Pathology, has published recommendations for the interpretation of these variants, ${ }^{9}$ and functional studies such as those performed by Shoemark and colleagues ${ }^{2}$ are considered a powerful tool in support of pathogenicity. The genetic diagnosis of PCD is especially challenging, as mutations in 39 genes have been implicated and this number continues to grow. ${ }^{10-15}$ The majority of the published literature describes PCD cases with two loss-of-function (truncating and splice site) pathogenic variants, raising the question of missing genetic diagnoses due to the interpretive limitations of missense, in-frame and close to the splice-site intronic variants. These latter variants also pose a dilemma for reporting of genetic results in the clinical setting. Various strategies including computational prediction algorithms may aid with interpretation, but often provide conflicting information. Functional studies such as described in this manuscript are a step in the right direction but are cost, time and labour intensive. The extensive genetic heterogeneity of PCD (and other Mendelian disorders) will continue to generate numerous VUSs. One possible way forward in elucidating their pathogenicity is to develop high throughput disease-specific functional assays, such as the recently described multiplexed assay for variant effect. ${ }^{16}$

Contributors All authors contributed to the writing of this editorial. MR organised the team and outline and contributed the content on PCD diagnosis. MAZ contributed the content on variants of uncertain significance. LEO contributed the content on functional studies. All authors reviewed and approved the final version.

Funding Supported by National Institutes of Health grants 5U54HL096458-06 (MAZ and MR), 5R01HL071798 (MAZ and LEO), 5R01HL117836-04-05 (LEO and MAZ).

Disclaimer The content is solely the responsibility of the authors and does not necessarily represent the official view of the NIH.

Competing interests None declared.

Provenance and peer review Commissioned; externally peer reviewed.

(C) Article author(s) (or their employer(s) unless otherwise stated in the text of the article) 2018. All rights reserved. No commercial use is permitted unless otherwise expressly granted.

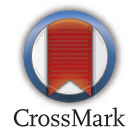

To cite Rosenfeld M, Ostrowski LE, Zariwala MA. Thorax 2018;73:101-102.

Published Online First 13 November 2017

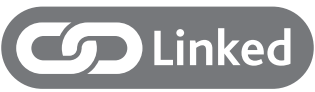

- http://dx.doi.org/10.1136/thoraxjnl-2017-209999

Thorax 2018;73:101-102.

doi:10.1136/thoraxjnl-2017-210776

\section{REFERENCES}

1 O'Callaghan C, Chetcuti P, Moya E. High prevalence of primary ciliary dyskinesia in a British Asian population. Arch Dis Child 2010;95:51-2.

2 Shoemark A, Moya E, Hirst RA, et al. High prevalence of CCDC103 p. His154Pro mutation causing primary ciliary dyskinesia disrupts protein oligomerisation and is associated with normal diagnostic investigations. Thorax 2018;73:157-66.

3 Ferkol TW, Puffenberger EG, Lie H, et al. Primary ciliary dyskinesia-causing mutations in Amish and Mennonite communities. J Pediatr 2013;163:383-7.

4 Panizzi JR, Becker-Heck A, Castleman VH, et al. CCDC103 mutations cause primary ciliary dyskinesia by disrupting assembly of ciliary dynein arms. Nat Genet 2012:44:714-9.

5 Zariwala MA, Knowles MR, Leigh MW, et al. Primary Ciliary Dyskinesia. In: Adam MP, Ardinger HH, Pagon RA, eds. GeneReviews(R). Seattle (WA): University of Washington.

6 Shapiro AJ, Josephson M, Rosenfeld M, et al. Accuracy of nasal nitric oxide measurement as a diagnostic test for primary ciliary dyskinesia. A systematic review and meta-analysis. Ann Am Thorac Soc 2017;14:1184-96.

7 Leigh MW, Ferkol TW, Davis SD, et al. Clinical features and associated likelihood of primary ciliary dyskinesia in children and adolescents. Ann Am Thorac Soc 2016;13:1305-13.

8 King SM, Patel-King RS. The oligomeric outer dynein arm assembly factor CCDC103 is tightly integrated within the ciliary axoneme and exhibits periodic binding to microtubules. I Biol Chem 2015;290:7388-401.

9 Richards S, Aziz N, Bale S, et al. Standards and guidelines for the interpretation of sequence variants: a joint consensus recommendation of the american college of medical genetics and genomics and the association for molecular pathology. Genet Med 2015;17:405-23.

10 Knowles MR, Zariwala M, Leigh M, et al. Primary ciliary dyskinesia. Clin Chest Med 2016;37:449-61.

11 Olcese C, Patel MP, Shoemark A, et al. X-linked primary ciliary dyskinesia due to mutations in the cytoplasmic axonemal dynein assembly factor PIH1D3. Nat Commun 2017:8:14279.

12 Paff T, Loges NT, Aprea I, et al. Mutations in PIH1D3 cause $X$-linked primary ciliary dyskinesia with outer and inner dynein arm defects. Am J Hum Genet 2017;100:160-8.

13 Edelbusch C, Cindrić S, Dougherty GW, et al. Mutation of serine/threonine protein kinase 36 (STK36) causes primary ciliary dyskinesia with a central pair defect. Hum Mutat 2017;38:964-9.

14 El Khouri E, Thomas L, Jeanson L, et al. Mutations in DNAJB13, encoding an HSP40 family member, cause primary ciliary dyskinesia and male infertility. Am J Hum Genet 2016;99:489-500.

15 Wallmeier J, Shiratori H, Dougherty GW, et al. TTC25 deficiency results in defects of the outer dynein arm docking machinery and primary ciliary dyskinesia with left-right body asymmetry randomization. Am J Hum Genet 2016;99:460-9.

16 Starita LM, Ahituv N, Dunham MJ, et al. Variant interpretation: functional assays to the rescue. Am J Hum Genet 2017;101:315-25. 